\title{
A IMPORTÂNCIA DA ANUSCOPIA DE ALTA RESOLUÇÃO PARA O DIAGNÓSTICO DO PAPILOMAVÍRUS HUMANO ANORRETAL NA FORMA SUBCLÍNICA, DAS LESÕES ANAIS INTRAEPITELIAIS E DO CARCINOMA "IN SITU”ANAL
}

\author{
IMPORTANCE OF HIGH RESOLUTION ANUSCOPY FOR THE DIAGNOSIS OF \\ SUBCLINICAL ANORECTAL HUMAN PAPILLOMAVIRUS, ANAL \\ INTRAEPITHELIAL NEOPLASIA AND ANAL CARCINOMA "IN SITU"
}

\author{
João Carlos Magi , TCBC-SP ${ }^{1}$ \\ Marcos Ricardo da Silva Rodrigues ${ }^{2}$ \\ Wladimyr Dias Moreno ${ }^{2}$ \\ João Baptista de Paula Fraga ${ }^{2}$ \\ Anderson da Costa Lino Costa ${ }^{3}$ \\ Galdino José Sitônio Formiga, ACBC-SP ${ }^{4}$
}

\begin{abstract}
RESUMO: Objetivo: A doença causada pelo Papilomavírus Humano (HPV) tem alta incidência na população e é considerada pré-neoplásica. O objetivo deste trabalho é estudar um método que detecte o HPV anorretal na sua forma subclínica e suas alterações neoplásicas, a incidência em pessoas com até três parceiros sexuais por ano e o resultado do tratamento com o uso de podofilina, ácido tricloroacético, eletrocauterização e ressecção local. Método: Vinte e dois pacientes foram submetidos ao exame de anuscopia de alta resolução. Os pacientes foram divididos em três grupos: o primeiro, formado por dez pacientes com prurido anal persistente mesmo após eliminadas causas como diabetes, doenças orificiais, outras doenças sexualmente transmissíveis, verminoses e submetidos a cuidados locais e dietéticos; o segundo e o terceiro, formados por seis pacientes com condiloma anal e seis pacientes com HPV genital respectivamente, ambos com alta do tratamento há mais de seis meses. Nenhum deles apresentava manifestação clínica da doença no momento do exame. Os casos com resultado positivo foram tratados com podofilina $25 \%$ e ácido tricloroacético, e os com carcinoma "in situ" com ressecção local e eletrocauterização da lesão. Resultados: O exame de anuscopia de alta resolução proporcionou a colheita dirigida do material nas áreas positivas, cujo resultado anátomo-patológico foi positivo para $\mathrm{HPV}$ em $100 \%$ dos pacientes, sendo dois deles com carcinoma "in situ" e oito com lesões intraepiteliais concomitantes. Apenas 9\% dos pacientes, tinham mais de três parceiros sexuais por ano. Todos os pacientes tratados com podofilina e ácido tricloroacético mantiveram as atipias coilocitóticas no controle após seis meses do início do tratamento. A ressecção local e eletrocauterização nos pacientes com carcinoma "in situ" possibilitou o desaparecimento da neoplasia em todos, mas manteve as atipias coilocitóticas. Conclusões: O exame de anuscopia de alta resolução possibilitou o diagnóstico de HPV anorretal na forma subclínica e suas alterações neoplásicas em $100 \%$ dos pacientes. O uso de podofilina e ácido tricloroacético não foi eficiente na erradicação das alterações anátomopatológicas decorrentes do HPV subclínico anal, no controle após seis meses do início do tratamento. A ressecção local e eletrocauterização dos casos de carcinoma "in situ” foi efetiva para erradicar a neoplasia , no entanto, com manutenção das atipias coilocitóticas.
\end{abstract}

Descritores: Papiloma; Carcinoma “in situ”; Ânus; Neoplasias do ânus; Diagnóstico.

1. Médico do Serviço de Coloproctologia do Hospital Heliópolis

2. Residente do Serviço de Coloproctologia do Hospital Heliópolis

3. Médico do Serviço de Anatomia Patológica do Hospital Heliópolis

4. Chefe do Serviço de Coloproctologia do Hospital Heliópolis

Trabalho realizado no Serviço de Coloproctologia do Hospital Heliópolis - São Paulo - SP. 


\section{INTRODUÇÃO}

A incidência da infecção causada pelo Papilomavírus Humano está crescendo nas últimas décadas, e é a doença viral sexualmente transmissível mais comum atualmente. Ela é também considerada uma lesão pré-neoplásica. ${ }^{1-8}$

A forma subclínica do HPV é a manifestação mais comum. Sua localização predominante é no ânus, vulva, vagina, colo uterino, pênis e cavidade oral, e o diagnóstico é feito pelo exame anátomo-patológico, quando encontramos atipias coilocitóticas, caracterizadas por células escamosas ou superficiais com grande cavitação, de bordos bem demarcados em torno de um núcleo atípico, sendo comum a binucleação ou multinucleação, cromatina nuclear densa e opaca ou granular, sempre hipercromática, citoplasma condensado em uma faixa na periferia celular, com coloração variando de eosinofílica a basofílica. O PCR (polymerase chain reaction), hibridização "in situ" e captura híbrida, possibilitam o diagnóstico das cepas virais. $\mathrm{O}$ exame anátomo-patológico, além de ter baixo custo também possibilita o diagnóstico do carcinoma e das lesões intraepiteliais anais. Todavia, é necessário que o material seja colhido em áreas comprometidas, e isto é possível corando-se a região com determinadas substâncias e examinando-a com lentes de aumento. $4,6,9-13$

O diagnóstico e tratamento das lesões intraepiteliais anais, do carcinoma "in situ" anal e da forma subclínica do HPV anorretal ainda é precário. Isto se deve, principalmente, por não ser usual entre os especialistas, a utilização do exame de anuscopia de alta resolução com colheita dirigida de material e uso de substâncias eficazes no tratamento local. ${ }^{11,14}$

Este trabalho tem por objetivo descrever a aplicação deste exame no diagnóstico e tratamento de casos de infecção por HPV, além de considerações sobre hábitos sexuais do grupo estudado.

$\mathrm{O}$ projeto de pesquisa deste trabalho registrado no Comitê de Ética em Pesquisa do Hospital Heliópolis sob número 185, foi aprovado em 10 de setembro de 2002.

\section{MÉTODO}

Vinte e dois pacientes foram submetidos a exame de anuscopia de alta resolução para diagnóstico de HPV anorretal na forma subclínica, lesões intraepiteliais e carcinoma "in situ" do canal anal.
Dezenove pacientes eram do sexo masculino e três do sexo feminino. A média de idade das mulheres foi 47 anos (40-57) e dos homens 42 anos (23-61).

O estudo prospectivo desenvolveu-se no Serviço de Coloproctologia do Hospital Heliópolis seguindo protocolo de diagnóstico e tratamento da doença.

Foram estudados seis pacientes tratados há mais de seis meses de HPV genital, seis pacientes tratados há mais de um ano de condiloma anal e dez pacientes com prurido anal persistente, após afastadas causas como diabetes, outras doenças sexualmente transmissíveis, verminoses, doenças orificiais, além de cuidados dietéticos e higiênicos locais como banhos de assento e o não uso de papel higiênico. Foram excluídos do estudo pacientes com lesões macroscópicas de HPV.

Os pacientes foram submetidos a exame de anuscopia de alta resolução, que consistiu na palpação da região, visualização com lente de aumento de 16 vezes sem corantes e depois com corantes. Os corantes utilizados foram o ácido acético a $5 \%$ e depois o azul de toluidina. As áreas corante-positivas foram biopsiadas.

Os equipamentos, materiais e substâncias usados foram: anuscópio descartável, colposcópio, pinça de biópsia, agulha, seringa, ácido acético a 5\%, azul de toluidina, xilocaína gel e xilocaína a $2 \%$ líquida.

Os pacientes com HPV subclínico anal abaixo da linha pectínea foram tratados com podofilina vaselinada a $25 \%$ e acima da linha pectínea com ácido tricloroacético a $90 \%$ dirigido às áreas coradas. Novas biópsias dirigidas foram realizadas seis meses depois, a fim de verificar a eficácia do tratamento.

Os pacientes com carcinoma "in situ" foram submetidos a ressecção local e eletrocauterização sob anestesia.

\section{RESULTADOS}

As lesões encontradas em todos foram: máculas planas, consideradas lesões não salientes e coradas, maiores que um ponto e menores de $0,5 \mathrm{~cm}$; manchas planas, consideradas lesões não salientes e coradas maiores que $0,5 \mathrm{~cm}$. Dois casos com área de pontilhado e um caso com área de mosaico concomitantes. A imagem do mosaico e pontilhado são as mesmas encontradas no exame de colposcopia.

O exame após a coloração, resultou positivo em todos os pacientes, evidenciado por áreas 
esbranquiçadas (Figura 1) no epitélio do canal anal após uso de ácido acético e áreas com coloração azul em tom escuro após uso do corante azul de toluidina. O estudo anátomo patológico do material colhido por biópsia dirigida, mostrou atipias coilocitóticas em todos os casos (Figura 2).
Todas as mulheres eram casadas, com um parceiro sexual e negaram relações anais. Duas mulheres pertenciam ao grupo de HPV genital e uma ao grupo de prurido anal. Todas as mulheres eram HIV negativas e três homens HIV positivos, sendo que somente dois tiveram mais de três parceiros sexuais

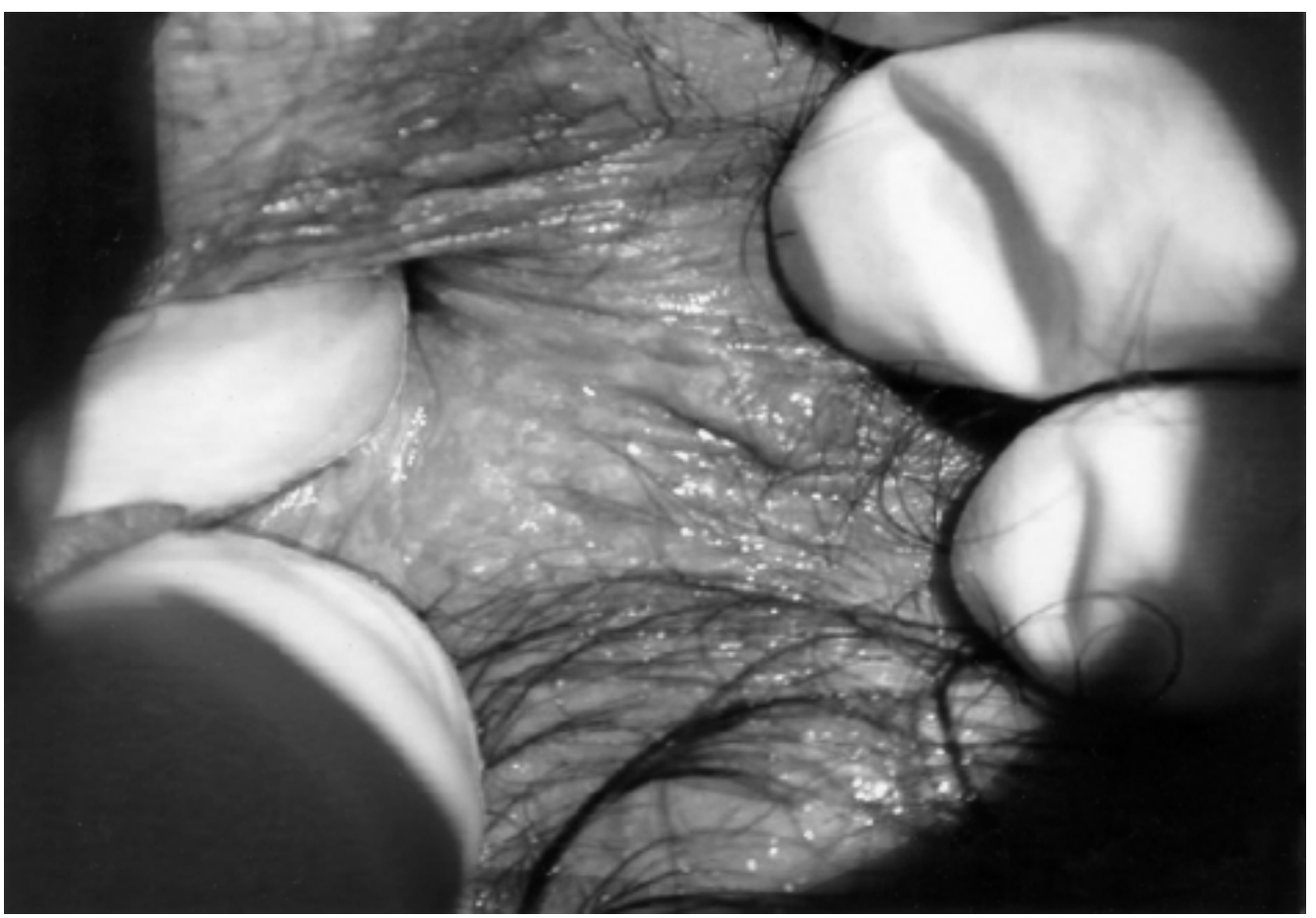

Figura 1 - Lesões aceto brancas.

por ano. Nove homens tiveram relações anais, dois na infância. Em todos os pacientes estudados, o uso de preservativo era feito de forma inadequada ou não era feito.

No grupo do HPV genital, composto de quatro homens e duas mulheres já tratados entre seis meses e trinta e três anos, um homem e uma mulher, apresentaram carcinoma "in situ" de canal anal e um homem apresentou lesão intraepitelial grau II (Tabela 1).

No grupo com prurido anal idiopático, composto de nove homens e uma mulher, quatro homens

Tabela 1 - Sub-grupo de Pacientes com Antecedentes de Condiloma Genital Tratado.

\begin{tabular}{lccccccl}
\hline Paciente & Sexo & Idade & HIV & Relação Anal & > 3 Parceiros ano HPV Subclínico & Lesão Intraepitelial \\
\hline 11 & M & 23 & - & Não & Não & + & Carcinoma "in situ" \\
12 & F & 57 & - & Não & Não & + & Carcinoma "in situ" \\
13 & F & 45 & - & Não & Não & + & - \\
14 & M & 37 & + & Sim & Sim & + & Grau II \\
15 & M & 53 & - & Não & Não & + & - \\
16 & M & 51 & - & Não & Não & + & - \\
\hline
\end{tabular}

$M=$ masculino $F=$ feminino . 


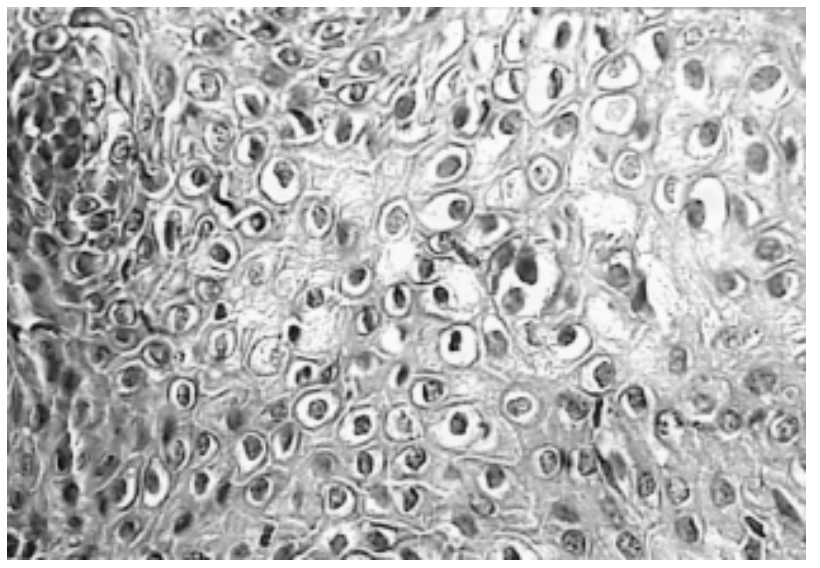

Figura 2 - Atipias coilocitóticas. Hematoxilina eosina 400x.

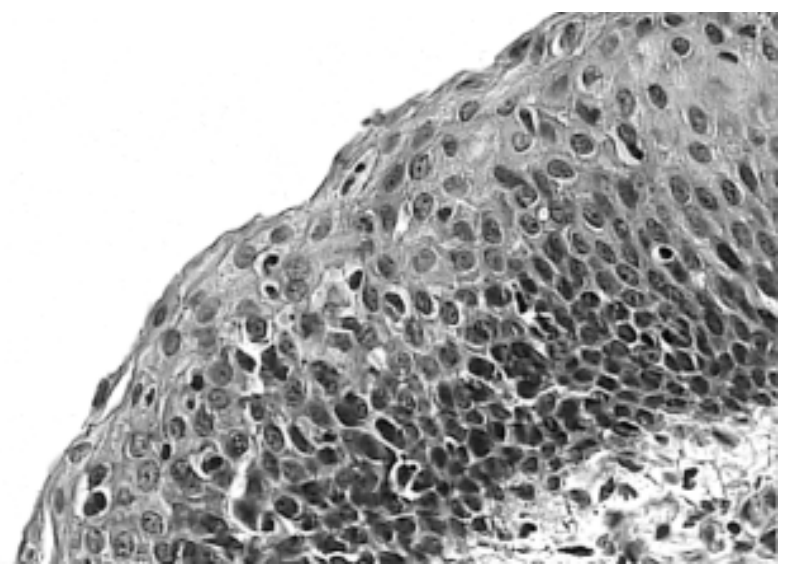

Figura 3 - Lesão intraepitelial grau II. Hematoxilina eosina 400x.

Tabela 2 - Sub-grupo de Pacientes com Sintoma de Prurido Anal Idiopático sem Antecedentes de HPV Genital ou Condiloma Anal.

\begin{tabular}{lccccccl}
\hline Paciente & Sexo & Idade & HIV & Relação Anal & > 3 Parceiros Ano HPV Subclínico Lesão Intraepitelial \\
\hline 1 & M & 32 & - & Sim & Não & + & Grau I \\
2 & M & 35 & - & Sim & Não & + & - \\
3 & M & 33 & - & Não & Não & + & - \\
4 & M & 48 & - & Não & Não & + & Grau I \\
5 & F & 40 & - & Não & Não & + & Grau I \\
6 & M & 58 & - & Sim & Não & + & Grau II \\
7 & M & 43 & - & Não & Não & + & Grau I \\
8 & M & 32 & - & Sim & Não & + & - \\
9 & M & 54 & - & Não & Não & + & - \\
10 & M & 42 & - & Não & Não & + & Grau I \\
\hline
\end{tabular}

M=masculino $F=$ feminino .

Tabela 3 - Sub-grupo de Pacientes com Antecedentes de Condiloma Anal Tratado.

\begin{tabular}{lccccccc}
\hline Paciente & Sexo & Idade & Hiv & Relação Anal & > 3 Parceiros ano HPV Subclínico Lesão Intraepitelial \\
\hline 17 & M & 37 & - & Não & Não & + & - \\
18 & M & 61 & - & Não & Não & + & - \\
19 & M & 27 & - & Sim & Não & + & - \\
20 & M & 53 & - & Sim & Não & + & - \\
21 & M & 42 & + & Sim & Não & + & - \\
22 & M & 49 & + & Sim & Sim & + & Grau II \\
\hline
\end{tabular}

$M=$ masculino, $F=$ feminino .

apresentaram lesão intraepitelial anal grau I, um homem lesão intraepitelial anal grau II (Figura 3) e a mulher lesão intraepitelial anal grau I (Tabela 2).

No grupo de condiloma anal, composto de seis homens já tratados entre um e dez anos, um apresentou lesão intraepitelial anal grau II (Tabela 3).
Dois homens HIV positivo, que mantinham relações anais e com mais de três parceiros por ano, apresentaram no anátomo-patológico lesão intraepitelial anal grau II; o outro homem HIV positivo que mantinha relações anais com menos de três parceiros sexuais por ano, apresentava apenas atipias coilocitóticas. 
O tratamento do carcinoma "in situ" foi eficiente na regressão da neoplasia mas houve manutenção das atipias coilocitoticas. As lesões intraepiteliais não foram erradicadas com o tratamento clínico.

Todos os pacientes estão em acompanhamento.

O colposcópio e a pinça de biópsia permitem ser reaproveitados para centenas de exames e o custo total das substâncias e material descartável usados é de aproximadamente um dólar por exame. A reação colateral foi apenas dor suportável, após o término do efeito da anestesia para a biópsia anal.

\section{DISCUSSÃO}

A doença causada pelo papilomavírus humano tem alta incidência na população, é considerada pré-neoplásica e é a doença viral sexualmente transmissível que mais cresce no mundo. ${ }^{1-4,6,7}$

O HPV subclínico é a forma de manifestação mais comum desta doença e o aumento de sua incidência se deve a fatores como hábitos do hospedeiro, interação hospedeiro-agente, métodos terapêutico/preventivos precários e aperfeiçoamento de métodos diagnósticos, como a colposcopia na área de ginecologia e peniscopia na área de urologia. ${ }^{1-3,7,9,15-17}$

O HPV acomete preferencialmente pele e mucosas e as regiões mais infectadas são o ânus, vulva, vagina, colo uterino, pênis e cavidade oral. 11

Há indícios que a região anorretal, tanto no homem quanto na mulher, tenha alta incidência comparada às outras regiões. Esta incidência elevada se deve à contaminação com vestimentas pela proximidade com os genitais, ambiente local abafado e úmido, relações anais e sensibilidade do anoderma pela doença. Ocorre que o diagnóstico do HPV anorretal subclínico ainda é subestimado, devido à pouca difusão do exame de anuscopia de alta resolução, e à maneira como são feitas a colposcopia, vulvoscopia e peniscopia. ${ }^{2,3,5,8,9}$

Este trabalho mostra a sensibilidade do exame para HPV subclínico, com 100\% de positividade no grupo estudado. Os resultados mostram que a região anorretal tem alta incidência de HPV subclínico e que o exame de anuscopia de alta resolução para diagnóstico destas lesões é eficiente, inclusive para detecção das lesões intraepiteliais anais e do carcinoma "in situ" anal. Autores como Jay et al alertam sobre as vantagens deste exame."

O exame de anuscopia de alta resolução possibilita visualizar lesões como pontilhado e mosaico, também encontradas na colposcopia, e manchas e máculas, como descritas neste trabalho, comuns na colposcopia, peniscopia e vulvoscopia.

Outro aspecto interessante deste exame é sua viabilidade, devido ao seu baixo custo, fácil manuseio, poucos efeitos colaterais e alta sensibilidade.

O tratamento desta doença ainda é precário. A cauterização é traumática ao paciente e apesar de eficiente para involuir macroscopicamente o condiloma ou forma clínica de HPV, é pouco eficiente no tratamento do HPV subclínico, como observamos após seis meses no controle do tratamento. Isto se deve, não só pela extensão das áreas infectadas, mas, principalmente, por ter o vírus tropismo pelas células da camada basal, dificultando o efeito da cauterização, que atinge predominantemente as camadas superficiais da epiderme. Alguns autores preconizam a excisão local e a cauterização profunda nos casos de carcinoma "in situ", pela possibilidade de evoluírem para carcinoma invasivo de canal anal. Os casos tratados desta maneira no grupo estudado resultaram eficientes na regressão do carcinoma "in situ", mas com manutenção das atipias coilocitóticas. O exame de anuscopia de alta resolução possibilita o desenho da área doente para o tratamento dirigido. $\mathrm{O}$ desenvolvimento de vacinas é também uma esperança no combate a esta doença. ${ }^{2,4,6,14,18}$

A associação desta doença com neoplasia tem causas multifatoriais, como falhas na imunidade do hospedeiro e virulência da cepa do agente. ${ }^{1,3,7,16}$

Apenas dois homens do grupo estudado tinham três parceiros sexuais ou mais por ano, o que demonstra que esta doença não acomete apenas pessoas com comportamento sexual de risco. Este dado, associado à possibilidade de contágio não sexual, e a doença poder se manter na forma latente e imperceptível durante anos, explicam a sua alta incidência em pessoas de hábitos comuns, fato este constatado no grupo de dez pacientes que procuraram o atendimento especializado com queixa única de prurido anal, sem antecedentes de HPV genital ou condiloma anal. $^{3,8,16}$ 


\begin{abstract}
Background: Human Papilloma Virus (HPV) infection has a high incidence in the population and is considered a pre malignant condition. The purpose of this study is to analyze a method of detection of subclinic anorectal HPV and its neoplasic changes; the incidence in people with more than three sexual partners a year and the results of the treatment with podofilin, trichloroacetic acid, electrocauterization and local resection. Methods: Twenty two patients were divided into three groups, and submitted to high resolution anuscopy. The first group included ten patients with persistent anal pruritus, in whom other causes were eliminated such as diabetes, orificial diseases, other sexual transmitted diseases, verminosis and were instructed about dietary and local care; the second and third groups included six patients treated for anal condylomata and six patients treated for genital HPV, respectively, for at least six months. None of them had clinical manifestations of the disease. Positive cases were treated with podofilin $25 \%$ and trichloroacetic acid, and carcinoma "in situ” cases were resected and electrocauterized. Results: Anuscopy provided guided biopsies in positive areas, which anatomopathologic results were positive for HPV infection in $100 \%$ of the patients, including two cases of carcinoma "in situ" and eight associated intraepithelial lesions. Only nine percent of these patients had more than three sexual partners per year. The koilocytotic atypia persisted in all patients treated with podofilin and trichloroacetic acid; local resection and electrocauterization of the carcinoma "in situ" resulted in total remission of the neoplasia but no eradication of the koilocytotic atypia. Conclusion: High resolution anuscopy provided diagnosis of subclinical HPV and its neoplastic changes in 100\% of the patients. Only nine percent had more than three sexual partners per year. Podofilin and trichloroacetic acid treatment was not effective to eradicate the anatomopathologic changes caused by subclinical HPV. The local resection and electrocauterization of the carcinoma "in situ" were effective, although, koilocytotic atypia was maintained.
\end{abstract}

Key Words: Papilloma; Carcinoma “in situ”; Anus; Anus neoplasms; Diagnosis.

\section{REFERÊNCIAS}

1. Critchlow CW, Hawes SE, Kuypers JM, et al. - Effect of HIV infection on the natural history of anal human papillomavirus infection. AIDS, 1998, 12(10):1177-1184.

2. Daling JR, Weiss NS, Hislop TG, et al. - Sexual practices, sexually transmitted diseases, and the incidence of anal cancer. N Engl J Méd, 1987, 317(16): 973-977.

3. Goldstone SE, Winkler B, Ufford LJ, et al. - High prevalence of anal squamous intraepithelial lesions and squamous-cell carcinoma in men who have sex whith men as seen in a surgical pratice. Dis Colon Rectum, 2001, 44(3): 690-698.

4. Nadal CRM. Evolução pós operatória dos condilomas acuminados perianais em doentes HIV positivos. Dissertação ( Doutorado em Medicna). São Paulo. Faculdade de Ciências Médicas da Santa Casa de São Paulo, 2000 .

5. Nadal SR, Manzione CR, Horta SHC, et al. - Tratamento tópico dos condilomas acuminados perianais em doentes HIV positivos. Rev Bras Coloproctol, 1999, 19(2):79-82.

6. Palefsky JM, Holly EA, Gonzales J, et al. - Detection of human papillomavirus DNA in anal intraepithelial neoplasia and anal cancer. Cancer Res, 1991, 51(3):10141019.
7. Palefsky JM, Holly EA, Ralston ML, et al. - High incidence of anal high-grade squamous intra-epithelial lesions among HIV-positive and HIV-negative homosexual and bisexual men. AIDS, 1998, 12(5): 495-503.

8. Ramanujam PS, Venkatesh KS, Co Barnett T, et al. - Study of human papillomavirus infection in patients with anal squamous carcinoma. Dis Colon Rectum, 1996,39(1):37-39.

9. Jay N, Berry JM, Hogeboom CJ, et al. - Colposcopic appearance of anal squamous intraepithelial lesions: relationship to histopathology. Dis Colon Rectum, 1997, 40(8):919-928.

10. Litle VR, Leavenworth JD, Darragh TM, et al. Angiogenesis, proliferation, and apoptosis in anal high-grade squamous intraepithelial lesions. Dis Colon Rectum, 2000, 43(3):346-352.

11. Magi JC, Magi DAS, Reche LMC, et al. - Anuscopia com exacerbação para diagnóstico de Papilomavírus Humano ano-retal na forma subclínica. Rev Bras Coloproct, 2002, 22(3):178-183.

12. Melbye M, Sprogel P - Aetiological parallel between anal cancer and cervical cancer. Lancet, 1991, 338(87968):657-659.

13. Pinto AP, Collaço LM - Revisão das alterações citomorfológicas da infecção pelo vírus do papiloma Humano em citologia cervicovaginal. J Bras Patol, 2001, 37(1):57-61. 
14. Chang GJ, Berry JM, Jay N, et al. - Surgical treatment of high-grade anal squamous intraepithelial lesions: a prospective study. Dis Colon Rectum, 2002, 45(4):453458.

15. Calore EE, Manzione CR, Nadal SR, et al. - Avaliação das causas de recidiva dos condilomas e neoplasias intraepiteliais anais na SIDA pela expressão do KI-67. Rev Bras Coloproct, 1999, 19 (supl):67.

16. Palefsky JM, Gonzales J, Greenblatt RM, et al. - Anal intraepithelial neoplasia and anal papillomavirus infection among homosexual males with group IV HIV disease. JAMA, 1990, 263(21):2911-2916.
17. Ranki A, Lassus J, Niemi KM - Relation of p53 tumor suppressor protein expression to human papillomavirus (HPV) DNA and to cellular atypia in male genital warts and in premalignant lesions. Acta Derm Venereol, 1995, 75(3):180-186.

18. Koutsky LA, Ault KA, Wheeler CM, et al. - A controlled trial of a human papillomavirus type 16 vaccine. N Eng J Med, 2002, 347(21):1645-1651.

Endereço para correspondência:

Dr. João Carlos Magi

Rua Paraiba 717 - Centro

CEP: 09521-070 - São Caetano do Sul - SP 